\title{
A pilot study of coronary angioplasty in outpatients
}

Gerrit J Laarman, Ferdinand Kiemeneij, L Ron van der Wieken, Jan G P Tijssen, Jo S M Suwarganda, Ton Slagboom

\begin{abstract}
Background-Is it safe to discharge patients from hospital on the same day as percutaneous transluminal coronary angioplasty (PTCA)? The hypothesis tested was that careful pre and post angioplasty selection of patients can identify a group that is at very low risk of postprocedural complications and that these patients may be discharged on the day of the procedure.

Methods -63 patients undergoing limited risk coronary angioplasty of 72 lesions were studied. So that patients would be able to walk soon after PTCA miniature equipment (6 French catheters and balloon-on-a-wire devices) was passed percutaneously through the right brachial artery. After coronary angioplasty patients with angiographic evidence of dissection and/or thrombus and with complications were assigned to an inpatient group and those in whom PTCA had achieved a good angiographic result were assigned to an outpatient group.
\end{abstract}

Results-Two patients were excluded because the brachial approach failed, leaving 61 patients (70 lesions). After PTCA 50 patients (82\%) with 57 lesions $(81 \%)$ attempted were assigned to the outpatient group. No cardiac complication occurred in this subset $(0 \% ; 95 \%$ confidence interval 0 to $7 \%$ ). Eleven patients $(18 \%)$, in whom 13 lesions $(19 \%)$ were attempted, were assigned to the inpatient group. Three of these patients (27\%; $95 \%$ confidence interval 6 to $61 \%$ ) had cardiac complications. Two patients needed local surgical repair after catheterisation of the brachial artery; one had a haematoma and one had a false aneurysm.

Conclusions-Coronary angioplasty with miniature equipment passed through the brachial artery was a safe procedure with a high initial success rate. The results of this pilot trial suggest that with careful selection of patients before and after angioplasty PTCA can be performed safely in outpatients.

(Br Heart $\mathcal{f ~ 1 9 9 4 ; 7 2 : 1 2 - 1 5 ) ~}$

Demand for diagnostic cardiac catheterisation and percutaneous transluminal coronary angioplasty (PTCA) is growing in the face of considerable constraints on resources. The hospital stay for these procedures has been reduced in recent years ${ }^{1}$ and diagnostic cardiac catheterisation is now widely applied as an outpatient procedure, with similar complication rates as in-hospital procedures. ${ }^{2}$ Same day discharge after PTCA is compromised by subacute vessel closure with subsequent infarction ${ }^{3}$ and because patients are immobilised for several hours after large bore sheaths are removed from the femoral artery.

The present prospective pilot study was conducted to test the hypothesis that with careful selection before and after angioplasty a subgroup of patients can be identified that is at very low risk of postprocedural lifethreatening complications and that can be discharged very early (same day) after the procedure. To allow patients to walk immediately after PTCA we used miniature angioplasty equipment and the percutaneous brachial approach.

\section{Patients and methods}

This investigation was designed as an open and prospective pilot study. The study group consisted of selected patients scheduled for elective PTCA for disabling angina pectoris between March 1991 and June 1992. All procedures were performed with surgical standby.

\section{OUTPATIENT MODEL}

Pre-PTCA selection

We selected patients at limited risk from PTCA. They had stable angina pectoris, suitable (non-complex) lesion morphology, a limited region at risk (to prevent haemodynamic instability and a large infarction in the event of late closure of the vessel). Hence we attempted lesions in side branches of major coronary arteries, distal coronary arteries, and major coronary arteries supplying partially infarcted myocardial regions or regions that were also supplied by well developed collaterals and lesions in vessels that had a diameter of $\leqslant 3.0 \mathrm{~mm}$. We excluded patients with renal insufficiency or uncontrolled hypertension.

\section{Post-PTCA triage}

Patients were observed for two hours close to the catheterisation laboratory. Then they were assigned either to an inpatient group or toan outpatient group. The group selected was recorded immediately. Patients with angiographic evidence of dissection or thrombus or both and patients with periprocedural 
Table 1 Clinical characteristics

\begin{tabular}{ll}
\hline Variable & No \\
\hline No of patients & 63 \\
Age (mean (SD)) & $59(10)$ y \\
Sex & 48 male \\
& $(76 \%)$
\end{tabular}
Angina pectoris
NYHA class:

$\begin{array}{lc}\text { NYHA class: } & 1(2) \\ \text { I } & 16(25) \\ \text { II } & 33(52) \\ \text { III } & 13(21) \\ \text { IV } & 40(63) \\ \text { Aspirin } & 9(14) \\ \text { Coumadines } & 4(6) \\ \text { Dipyridamole } & 70 \\ \text { Previous infarction } & 30(48) \\ \text { Previous CABG } & 7(11) \\ \text { Previous PTCA } & 11(17) \\ \text { Peripheral disease } & 12(19) \\ \text { Hypertension } & 16(25) \\ \text { Diabetes mellitus } & 6(10) \\ \text { Single vessel } & 30(48) \\ \text { disease } & \end{array}$

Multivessel disease $33(52)$

NYHA, according to the New York Heart Association Classification; CABG, coronary artery bypass graft surgery

Table 2 Angiographic variables of target vessels.

\begin{tabular}{lc}
\hline Variable & $n(\%)$ \\
\hline RCA $^{\star}$ & $22(31)$ \\
LCX $^{\star}$ & $24(33)$ \\
LAD $^{\star}$ & $23(32)$ \\
Saphenous vein & $2(3)$ \\
$\quad$ graft & \\
Left internal & $1(1)$ \\
$\quad$ mammary artery & \\
Lesion type: & \\
A & $64(89)$ \\
B1 & $7(10)$ \\
B2 & $1(1)$ \\
C & $0(0)$ \\
\hline
\end{tabular}

RCA, right coronary artery; LCX, left circumflex coroLCX, left circumfiex coronary artery; LAD, left anterior descending coronary
artery; $\star$ Major coronary artery; Major coronary

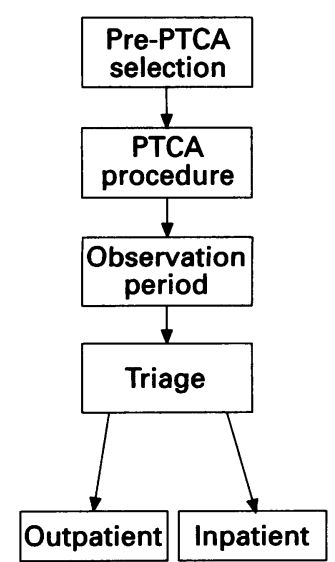

Figure Study protocol. complications were assigned to the inpatient group. Those in whom PTCA was successful (that is, diameter stenosis $<50 \%$ with absence of recurrent angina pectoris, acute occlusion, repeat angioplasty, urgent bypass surgery, myocardial infarction, or death) with a good angiographic result were assigned to the outpatient group. Allocation to the outpatient and inpatient groups was at the discretion of the operator.

Because the risks of PTCA performed on an outpatient basis are ill defined we did not discharge patients from the hospital two hours after PTCA. Patients stayed overnight in the hospital without any monitoring. Before discharge the next morning they were examined by a trained physician.

\section{CORONARY ANGIOPLASTY}

Patients were admitted directly to the catheterisation laboratory. To allow patients to walk immediately after PTCA we used a percutaneous approach from the right brachial artery with miniature (6 French) equipment. Patients were informed about the study protocol and their consent was obtained. A soft-tipped 6 French catheter (BARD-USCI, Pro-Flo) was advanced through a 6 French $10 \mathrm{~cm}$ introduction sheath (Bard-USCI, Hemaquet II) and the coronary ostium was cannulated. At the time of this study only 6 French diagnostic catheters were available. Heparin (100 U/kg body weight) was given through the sheath. Cineangiography in multiple projections was performed with a Philips Digital Cardiac Imaging system. Coronary dilatation was performed with $2 \cdot 0,2 \cdot 5$, and $3 \cdot 0 \mathrm{~mm}$ balloon-ona-wire devices (BARD-USCI, Probe III), that can be used in conjunction with this 6 French diagnostic catheter. After dilatation the balloon catheter was left in situ and a follow up angiogram was performed. When the result appeared satisfactory the balloon catheter was pulled back and removed from the guiding catheter to allow optimal opacification of the dilated segment. Immediately after the procedure the introduction sheath was removed in all cases and manual compression was applied to the brachial artery. Heparin was not neutralised by protamine sulphate. Characteristics of the dilated vessel segment such as haziness, dissection, and side branch occlusion, were noted. We noted the duration of compression needed after sheath removal to stop bleeding. When there was evidence of dissection we maintained a partial thromboplastin time of 2-3 times baseline for 12-24 hours by extending the duration of heparin infusion.

Pre and post PTCA angiograms were separately analysed by two trained angiographers. The following features were recorded: type of lesion before PTCA (type A, B1, B2, and $C^{45}$ ), angiographic success of PTCA (diameter stenosis $<50 \%$ ), and evidence of dissection and/or thrombus. Visualisation of the PTCA segment with and without the balloon catheter in situ was graded as poor, adequate, or good.
Results

From March 1991 to June 19921050 PTCA procedures were performed in our department. Of these, 63 patients $(6 \%)$ were selected for the present study. This number was small because the inclusion criteria were rigid and the procedures were performed by only one operator (GJL). In the same period this operator performed 305 PTCA procedures. This series is not entirely consecutive, but this may not matter because the study was prospective. Table 1 shows the clinical characteristics of the patients and table 2 shows the angiographic variables.

In two patients the brachial approach was unsuccessful. In one patient spasm prevented puncture of the brachial artery and in another the coronary stenosis could not be reached by the balloon catheter. Both patients were successfully treated via the femoral route. These two patients were excluded from subsequent analysis. Seventy stenoses were attempted in the remaining 61 patients and PTCA failed in two patients. In one patient there was abrupt occlusion of a diagonal branch in the laboratory and this could not be recrossed with the guide wire. Because the jeopardised region was small this condition was accepted. The patient sustained an uncomplicated non- $Q$ wave myocardial infarction with a maximal creatine kinase value of $626 \mathrm{U} / 1$. In another patient multilesion PTCA was attempted and a lesion in a small posterolateral branch of the right coronary artery could not be crossed. No further attempts were made because the culprit lesion in this patient was successfully dilated. Coronary angioplasty was performed with a $2.0 \mathrm{~mm}$ balloon in nine segments, a 2.5 $\mathrm{mm}$ in 41 segments, and a $3.0 \mathrm{~mm}$ balloon in 20 segments. The mean maximal inflation pressure was $10.2 \mathrm{~atm}(\mathrm{SD}(2 \cdot 7))$.

During the 2 hour observation period two patients sustained acute occlusion within 30 minutes of leaving the catheterisation laboratory. Both patients underwent urgent repeat angioplasty via the femoral route, with good results. In one of these patients creatine kinase rose to $772 \mathrm{U} / \mathrm{l}$ but no new $\mathrm{Q}$ waves developed.

Visualisation of the PTCA segment with the balloon catheter in situ was graded as poor in $2(3 \%)$, moderate in $5(7 \%)$, and good in $63(90 \%)$ of the cases. After the balloon catheter was retrieved, these figures were 2 $(3 \%), 3(4 \%)$, and $65(93 \%)$ respectively.

\section{POST-PTCA ASSIGNMENT}

Fifty patients (82\%) with 57 lesions (81\%) attempted were assigned to the outpatient category. None of these patients had a cardiac complication $(0 \% ; 95 \%$ confidence interval $0 \%$ to $7 \%$ ). Eleven patients $(18 \%)$ in whom 13 lesions $(19 \%)$ were attempted were assigned to the inpatient group, including those with abrupt occlusion described above. All of these patients were given intravenous heparin for 12-24 hours because there was angiographic evidence of dissection or thrombus or both. Three inpatients (27\%; 95\% confidence interval $6 \%$ to $61 \%$ ) had cardiac 
complications. Two patients sustained acute occlusion within an hour of the observation period and repeat PTCA was successful without evidence of myocardial infarction. One patient had acute occlusion 20 hours after the procedure. Because the result of urgent repeat PTCA via the femoral approach was unsatisfactory, a Palmaz-Schatz intracoronary stent was implanted. Creatine kinase measurements remained within normal limits and the clinical course was uneventful. The occurrence of acute occlusion in the whole study group was not related to sex (female $13 \% v$ male $8 \%$ ) or to the extent of coronary artery disease (multivessel disease $9 \% v$ single vessel disease $10 \%)$.

PERCUTANEOUS BRACHIAL APPROACH

During the procedure no patient experienced ischaemic symptoms in the right forearm. The mean (SD) compression time until haemostasis was 14(9) min (range 4-60 min). After the procedure a haematoma was found near the puncture site in four patients $(7 \%)$. In one case $(2 \%)$ a false aneurysm was suspected. This was confirmed by echo-Doppler examination. Two patients had surgical repair, one for haematoma and one for a false aneurysm. Medial nerve injury did not occur. Before discharge all patients had good pulsations in the radial and brachial arteries.

\section{Discussion}

The considerable expansion of PTCA has taken up a substantial part of the admission capacity and financial resources of the cardiology department of large referral centres. Many centres are reducing the hospital stay for uncomplicated procedures. The main obstacles to same day discharge after PTCA are delayed ambulation after catheterisation of the femoral artery, especially when large bore ( 8 French) introducer sheaths are used, with the risks of bleeding complications and acute occlusion of the dilated segment.

The advantages of the use of miniature equipment are the small arterial puncture site, which is likely to need shorter compression and to facilitate early ambulation, and less compromised blood flow to the extremities when small arteries (brachial or radial arteries) are cannulated or when there is obstructive peripheral vascular disease. ${ }^{6-9}$ Other possible advantages are: less pressure damping and injury to the coronary artery ostia caused by the catheter, especially in a small or diseased main stem or right coronary artery ostium. ${ }^{7}$ These advantages should increase patient comfort and reduce hospital stay.

A potential problem associated with miniature equipment is unsatisfactory vessel visualisation. In our series visualisation was adequate in more than $95 \%$ of instances. Small bore catheters preclude the use of bailout equipment such as perfusion balloons or stents and multiple balloon catheters; in addition their use is associated with inferior backup, poor catheter seating, and inadequate torque control. ${ }^{6-8}$ Developments in small bore guiding catheters of 6 French with better performance and larger internal diameter will overcome some of these limitations. ${ }^{9}$

Percutaneous entry of the brachial artery with small bore guiding catheters leaves the transfemoral approach available in the event of failure; it costs less and does not require complex bed transfers (because patients are ambulant immediately after PTCA); and it facilitates early hospital discharge, and thus makes outpatient PTCA a possibility. ${ }^{10}$ Acute occlusion is sometimes unpredictable: none the less, certain predictive variables have been recognised. These include unstable angina and complex lesion morphology. ${ }^{311} 12$ In some studies female sex and the presence of extensive coronary disease seemed to be of prognostic significance. ${ }^{11}$ But because female sex was not a strong independent risk marker in all studies of acute closure after PTCA ${ }^{312}$ we did not exclude women from this pilot study. Similarly we also included patients with multivessel coronary artery disease because de Feyter et al found that multivessel disease was of weak prognostic significance. ${ }^{3}$ de Feyter et al reported a probability range for acute occlusion of between $2 \%$ (no pre-PTCA predictive factors present) and $25 \%$ (all prePTCA predictive factors present). ${ }^{3}$ It is not known whether these predictors are of postprocedural significance when the initial PTCA result is good. In our study the occurrence of acute occlusion was not related to sex or to the extent of coronary artery disease. We included patients with multivessel or multilesion PTCA because the risk of abrupt occlusion was no higher than in PTCA of single vessels or single lesions. ${ }^{313}$ In the $1985-1986$ National Heart Lung and Blood Institute PTCA Registry study post-procedural predictive factors of acute occlusion were angiographic evidence of an intimal dissection and haziness of the dilated segment. ${ }^{12}$ The overall incidence of acute occlusion after the patient had left the catheterisation laboratory was less than $2 \% .^{312}$ Therefore, careful patient selection before PTCA (minimal prevalence of predictive variables) followed by post-PTCA assignment to a low risk patient subset (no angiographic signs of dissection or thrombus) will produce patient group at a very low risk of acute occlusion outside the laboratory. Little is known about the time course of acute occlusion after the patient has left the catheterisation laboratory because the low frequency of this complication precludes meaningful analysis. Post-procedural risks may be reduced further if the patient remains symptom free during an observation period in the catheterisation laboratory area. To take account of the infrequent complication of unexpected delayed closure, the selection of patients with a limited region at risk may lessen the risk of haemodynamic deterioration and subsequent large myocardial infarcts.

In this study the clinical course of all patients referred to the outpatient group was uneventful. The results $(95 \%$ confidence 
interval $0 \%$ to $7 \%$ ), however, do not preclude complication rates as high as $\mathbf{7 \%}$. Therefore, larger series of patients should be studied to identify the complication rate with greater precision. These 11 patients with evidence of dissection and/or periprocedural complications sustained acute occlusion after the 2 hour observation period. No emergency coronary bypass surgery was needed.

Though it is based on predetermined criteria, post-PTCA triage remains a subjective selection procedure. As the triage decision is based primarily on angiographic information, optimal imaging systems equipped with a digital facility are essential. In this pilot trial we used a conservative and restrictive approach. In addition to recommending the investigation of a larger series of patients in carefully designed outpatient protocols, we caution against using more liberal standards for selection of outpatient candidates at this stage because acute complications occurring outside the hospital will discredit outpatient PTCA. In addition, the results of our study do not imply that these PTCA procedures could have been performed without immediate surgical stand-by. PTCA was performed only if surgical stand-by was available, because in most patients serious complications would have required urgent surgery. Furthermore, outpatient PTCA should not be allowed to lead to under revascularisation.

We conclude that coronary angioplasty with miniature equipment via the brachial artery can be done safely with a high initial success rate. Our results suggest that with careful patient selection (before and after angioplasty) PTCA can safely be performed on an outpatient basis. Studies of a larger series of patients are needed to confirm the promising findings of this pilot trial.

1 Cragg DR, Friedman HZ, Almany SL, et al. Early hospital discharge after percutaneous transluminal coronary angioplasty. Am $\mathcal{F}$ Cardiol 1989;64:1270-4.

2 Block PC, Ockene I, Goldberg RJ, et al. A prospective randomized trial of outpatient versus inpatient cardiac catheterization. N Engl f Med 1988;319:1251-5.

3 de Feyter PJ, van den Brand M, Laarman GJ, van Domburg R, Serruys PW, Suryapranata H. Acute coronary occlusion during and after percutaneous transluminal coronary angioplasty: frequency, prediction, clinical course, management, and follow-up. Circulation
1991;83:927-86.

4 ACC/AHA Task Force Report. Guidelines for percutaneous transluminal coronary angioplasty. $f \mathrm{Am}$ Coll Cardiol 1988;12:529-35.

5 Ellis SG, Vandormael MG, Cowley MJ, DiSciascio G Delingonul U, Topol EJ, Bulle TM, and the Multivessel angioplasty Prognosis Study Group. Coronary morphologic and clinical determinants of procedural outcome logic and clinical determinants of procedural outcome
with angioplasty for multivessel coronary disease. with angioplasty for multivess

6 Moles VP, Meier B, Urban P, de la Serna F, Pande AK. Percutaneous transluminal coronary angioplasty through 4 French diagnostic catheters. Cathet Cardiovasc Diagn 1992;25:98-100.

7 Kern MJ, Talley JD, Deligonul U, et al. Preliminary experience with 5 and 6 French diagnostic catheters as guiding catheters for coronary angioplasty. Cathet Cardiovasc Diagn 1991;22:60-3.

8 Feldman R, Kaizer J, Standley M. Coronary angioplasty using new 6 French guiding catheters. Cathet Cardiovasc Diagn 1991;23:93-9.

9 Ueno K, Kotoo Y, Arai M, et al. Coronary angioplasty using an over-the-wire balloon catheter through a new 6 using an over-the-wire balloon catheter through a new 6 26:61-8.

10 Kamada RO, Fergusson DJG, Itagaki RK. Percutaneous entry of the brachial artery for transluminal coronary angioplasty. Cathet Cardiovasc Diagn 1988;15:132-3.

11 Ellis SG, Roubin GS, King SB III, et al. Angiographic and clinical predictors of acute closure after native vessel coronary angioplasty. Circulation 1988;77:372-9.

12 Detre KM, Holmes DR, Holubkov R, Cowley MJ, Bourassa MG, Faxon DP, Dorros GP, Bentivoglio LB, Kent KM, Myeler RK, coinvestigators of the NHLBI PTCA Registry. Incidence and consequences of periprocedural occlusion: The 1985-1986 NHLBI PTCA Registry. Circulation 1990;82:739-50.

13 Gaul G, Hollman J, Simpendorfer C, Franco I. Acute occlusion in multiple lesion coronary angioplasty. Frequency and management. F Am Coll Cardiol 1989; 13:283-8. 